\title{
Generation of Macroscopic Singlet States in a Cold Atomic Ensemble
}

\author{
N. Behbood, ${ }^{1,}{ }^{*}$ F. Martin Ciurana, ${ }^{1}$ G. Colangelo, ${ }^{1}$ M. Napolitano, ${ }^{1}$ Géza Tóth,${ }^{2,3,4}$ R. J. Sewell, ${ }^{1}$ and M. W. Mitchell ${ }^{1,5}$ \\ ${ }^{1}$ ICFO_Institut de Ciencies Fotoniques, Mediterranean Technology Park, 08860 Castelldefels (Barcelona), Spain \\ ${ }^{2}$ Department of Theoretical Physics, University of the Basque Country UPV/EHU, P.O. Box 644, E-48080 Bilbao, Spain \\ ${ }^{3}$ IKERBASQUE, Basque Foundation for Science, E-48011 Bilbao, Spain \\ ${ }^{4}$ Wigner Research Centre for Physics, Hungarian Academy of Sciences, P.O. Box 49, H-1525 Budapest, Hungary \\ ${ }^{5}$ ICREA—Institució Catalana de Recerca i Estudis Avançats, 08015 Barcelona, Spain
}

(Received 2 April 2014; published 25 August 2014)

\begin{abstract}
We report the generation of a macroscopic singlet state in a cold atomic sample via quantum nondemolition measurement-induced spin squeezing. We observe $3 \mathrm{~dB}$ of spin squeezing and detect entanglement with $5 \sigma$ statistical significance using a generalized spin-squeezing inequality. The degree of squeezing implies at least $50 \%$ of the atoms have formed singlets.
\end{abstract}

PACS numbers: 42.50.-p, 42.50.Ct, 42.50.Dv

Generating and detecting large-scale spin entanglement in many-body quantum systems is of fundamental interest $[1,2]$ and motivates many experiments with cold atoms [3-7] and ions [8]. For example, macroscopic singlet states appear as ground states of many fundamental spin models $[9,10]$ and even in quantum gravity calculations of black hole entropy [11]. Here, we report the production of a macroscopic spin singlet (MSS) in an atomic system using collective quantum nondemolition (QND) measurement [12-14] as a global entanglement generator.

QND measurement is a well-established technique for generating conditional spin squeezing in polarized atomic samples [15-21], where the state of the art is $10 \mathrm{~dB}$ of squeezing in a cavity-enhanced measurement [22]. In our experiment, we apply QND measurement techniques to an unpolarized sample. The QND measurement first generates large-scale atom-light entanglement by passing a macroscopic optical pulse through the entire ensemble. The optical pulse is then measured, transferring the entanglement onto the atoms and leaving them in an entangled state [23]. Subsequent measurements on the ensemble confirm the presence of a MSS with a singlet fraction of approximately $1 / 2$. Our techniques are closely related to proposals for using QND measurement to detect $[24,25]$ and generate [26] long-range correlations in quantum lattice gases and spinor condensates.

A MSS has a collective spin $\hat{\mathbf{F}}=\mathbf{0}$, where $\hat{\mathbf{F}} \equiv \sum_{i} \hat{\mathbf{f}}^{(i)}$ and $\hat{\mathbf{f}}^{(i)}$ is the spin of the $i$ th atom. This implies that fluctuations in the collective spin vanish, i.e., $\Delta \hat{\mathbf{F}}=\mathbf{0}$, suggesting that we can both produce and detect a macroscopic singlet via QND measurement-induced spin

Published by the American Physical Society under the terms of the Creative Commons Attribution 3.0 License. Further distribution of this work must maintain attribution to the author(s) and the published article's title, journal citation, and DOI. squeezing [23,26]. Indeed, it has been shown that a macroscopic spin singlet can be detected via the generalized spin-squeezing parameter

$$
\xi^{2}=\frac{\sum_{k}\left(\Delta \hat{F}_{k}\right)^{2}}{f N_{A}}
$$

where $\xi^{2}<1$ indicates spin squeezing in the sense of noise properties not producible by any separable state and thus detects entanglement among the atoms [23,27-31]. The standard quantum limit for unpolarized atoms is set by $\xi^{2}=1$, i.e., $\sum_{k}\left(\Delta \hat{F}_{k}\right)^{2}=f N_{A}$. The number of atoms that are at least pairwise entangled in such a squeezed state is lower bounded by $\left(1-\xi^{2}\right) N_{A}$ [23]. In the limit $\xi^{2} \rightarrow 0$, the macroscopic many-body state is a true spin singlet. Another criterion for detecting entanglement in nonpolarized states has recently been applied to Dicke-like spin states [32]. Our results complement recent work with quantum lattice gases $[3,5,7]$ and are analogous to the generation of macroscopic singlet Bell states with optical fields [33,34].

Since the collective spin obeys spin uncertainty relations $\left(\Delta \hat{F}_{i}\right)^{2}\left(\Delta \hat{F}_{j}\right)^{2} \geq\left|\left\langle\hat{F}_{k}\right\rangle\right|^{2} / 4$ (we take $\hbar=1$ throughout), squeezing all three spin components requires maintaining an unpolarized atomic sample with $\left\langle\hat{F}_{k}\right\rangle \simeq 0$. Our experiment starts from a thermal spin state (TSS), i.e., a completely mixed state described by a density matrix $R=\rho^{\otimes N_{A}}$, where $\rho=\frac{1}{3} \mathbb{1}_{3 \times 3}$ and $\mathbb{1}_{3 \times 3}$ is the identity matrix. This state has $\left\langle\hat{F}_{k}\right\rangle=0$ and $\left(\Delta \hat{F}_{k}\right)^{2}=(2 / 3) N_{A}$. It is symmetric under exchange of atoms and mixed at the level of each atom.

We probe the atoms via paramagnetic Faraday rotation using pulses of near-resonant propagating along the trap axis to give a high-sensitivity measurement of $\hat{F}_{z}$. The optical pulses are described by Stokes operators $S$, which obey $\left[\hat{S}_{x}, \hat{S}_{y}\right]=i \hat{S}_{z}$ and cyclic permutations. The input pulses are fully $\hat{S}_{x}$ polarized, i.e., with $\left\langle\hat{S}_{x}\right\rangle=N_{L} / 2$, where $N_{L}$ is the number of photons in the pulse. During a 
measurement pulse, the atoms and light interact via an effective Hamiltonian (see the Supplemental Material [35])

$$
\tau \hat{H}_{\mathrm{eff}}=G_{1} \hat{S}_{z} \hat{F}_{z},
$$

where $G_{1}$ is a coupling constant describing the vector light shift and $\tau$ is the pulse duration [36,37]. Equation (2) describes a QND measurement of $\hat{F}_{z}$, i.e., a measurement with no backaction on $\hat{F}_{z}$. We detect the output

$$
\hat{S}_{y}^{(\text {out })}=\hat{S}_{y}^{(\text {in })}+G_{1} \hat{S}_{x}^{(\text {in })} \hat{F}_{z}^{(\text {in })},
$$

which leads to measurement-induced conditional spin squeezing of the $\hat{F}_{z}$ component by a factor $1 /(1+\zeta)$, where $\zeta=\frac{2}{3} G_{1}^{2} N_{L} N_{A}$ is the signal-to-noise ratio (SNR) of the measurement [38].

To measure and squeeze the remaining spin components, we follow a stroboscopic probing strategy described in Refs. [39,40]. We apply a magnetic field along the [111] direction so that the collective atomic spin rotates $\hat{F}_{z} \rightarrow$ $\hat{F}_{x} \rightarrow \hat{F}_{y}$ during one Larmor precession cycle. We then time our probe pulses to probe the atoms at $T_{L} / 3$ intervals, allowing us to measure all three components of the collective spin in one Larmor period. Note that the probe duration $\tau \ll T_{L}$, so that we can neglect the rotation of the atomic spin during a probe pulse.

This measurement procedure respects the exchange symmetry of the input TSS and generates correlations among pairs of atoms independent of the distance between them, leading to large-scale entanglement of the atomic spins. The resulting state has $\left(1-\xi^{2}\right) N_{A}$ spins entangled in a MSS and $\xi^{2} N_{A}$ spin excitations (spinons).

Our experimental apparatus, illustrated in Fig. 1(a), is described in detail in Ref. [41]. In each cycle of the experiment, we trap up to $1.5 \times 10^{6}{ }^{87} \mathrm{Rb}$ atoms in a weakly focused single beam optical dipole trap. The atoms are laser cooled to a temperature of $20 \mu \mathrm{K}$ and optically pumped into the $f=1$ hyperfine ground state. A shotnoise-limited balanced polarimeter detects $\hat{S}_{y}^{\text {(out) }}$ while a reference detector before the atoms measures $\hat{S}_{x}^{(\text {in) }}$. The trap geometry produces a large atom-light interaction for light pulses propagating along the axis of the trap, quantified by the effective optical depth $d_{0}=\left(\sigma_{0} / A\right) N_{A}$, where $\sigma_{0}=$ $\lambda^{2} / \pi$ and $A=2.7 \times 10^{-9} \mathrm{~m}$ is the effective atom-light interaction area [41], giving $d_{0}=69.5$ with $N_{A}=1.5 \times$ $10^{6}$ atoms. We measure an atom-light coupling constant $G_{1}=9.0 \pm 0.1 \times 10^{-8} \mathrm{rad}$ per spin (see the Supplemental Material [35]). The measured sensitivity of the Faraday rotation probing is $\Delta F_{z}=515$ spins [12], allowing projection-noise-limited probing of an input TSS with $N_{A}>1.75 \times 10^{5}$ atoms.

The measurement sequence is illustrated in Figs. 1(b) and 1(c). For each measurement, the atoms are initially prepared in a TSS via repeated optical pumping of the
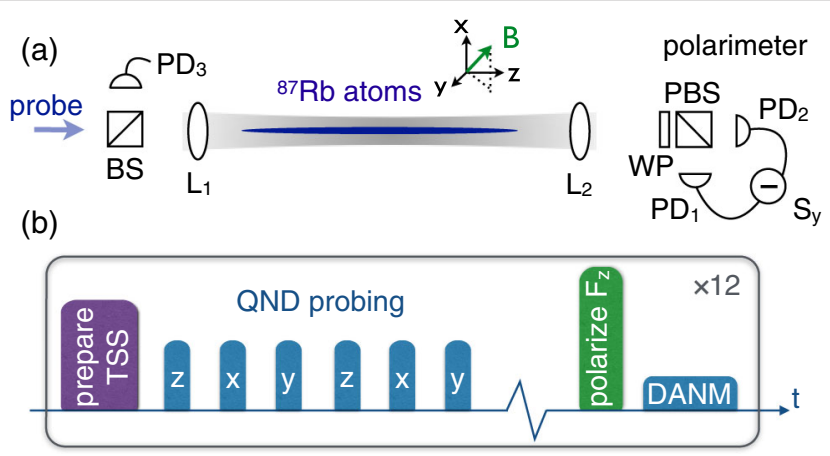

(c)

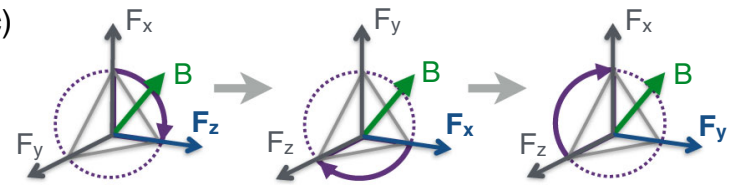

FIG. 1 (color online). (a) Experimental geometry. Nearresonant probe pulses pass through a cold cloud of ${ }^{87} \mathrm{Rb}$ atoms and experience a Faraday rotation by an angle proportional to the on-axis collective spin $\hat{F}_{z}$. The pulses are initially polarized with a maximal Stokes operator $\hat{S}_{x}$ recorded on a reference detector $\left(\mathrm{PD}_{3}\right)$. Rotation toward $\hat{S}_{y}$ is detected by a balanced polarimeter consisting of a wave plate (WP), polarizing beam splitter (PBS), and photodiodes $\left(\mathrm{PD}_{1,2}\right)$. (b) Pulse sequence: A first QND measurement measures the $\hat{F}_{z}$ angular momentum component of the input atomic state, and the second and third QND measurements in $1 / 3$ and $2 / 3$ of Larmor precession cycles measure $\hat{F}_{y}$ and $\hat{F}_{x}$, respectively. (c) $\hat{\mathbf{F}}$ precesses about a magnetic field (B) along the direction [111], making all components accessible to measurement via stroboscopic probing.

atoms between $f=1$ and $f=2$, as described in Ref. [12]. We then probe the atomic spins using a train of $\tau=1 \mu \mathrm{s}$ long pulses of linearly polarized light, detuned by $700 \mathrm{MHz}$ to red of the $f=1 \rightarrow f^{\prime}=0$ transition of the $D_{2}$ line. Each pulse contains on average $N_{L}=2.8 \times 10^{8}$ photons. To access also $\hat{F}_{x}$ and $\hat{F}_{y}$, we apply a magnetic field with a magnitude $B=16.9 \pm 0.1 \mathrm{mG}$ along the direction [111]. The atomic spins precess around this applied field with a Larmor period of $T_{L}=85 \mu \mathrm{s} \gg \tau$, and we probe the atoms at $T_{L} / 3=28.3 \mu \mathrm{s}$ intervals for two Larmor periods, allowing us to analyze the statistics of repeated QND measurements of the collective spin.

After the QND probing, the number of atoms $N_{A}$ is quantified via dispersive atom number measurement (DANM) [12,13] by applying a bias field $B_{z}=100 \mathrm{mG}$, optically pumping the atoms into $\left|f=1, m_{f}=1\right\rangle$ with circularly polarized light propagating along the trap axis resonant with the $f=1 \rightarrow f^{\prime}=1$ transition, and then probing with the Faraday rotation probe.

The sequence of state preparation, stroboscopic probing, and DANM is repeated 12 times per trap loading cycle. In each sequence, $\sim 15 \%$ of the atoms are lost, mainly during the state preparation, so that different values of $N_{A}$ are sampled during each loading cycle. At the end of each 

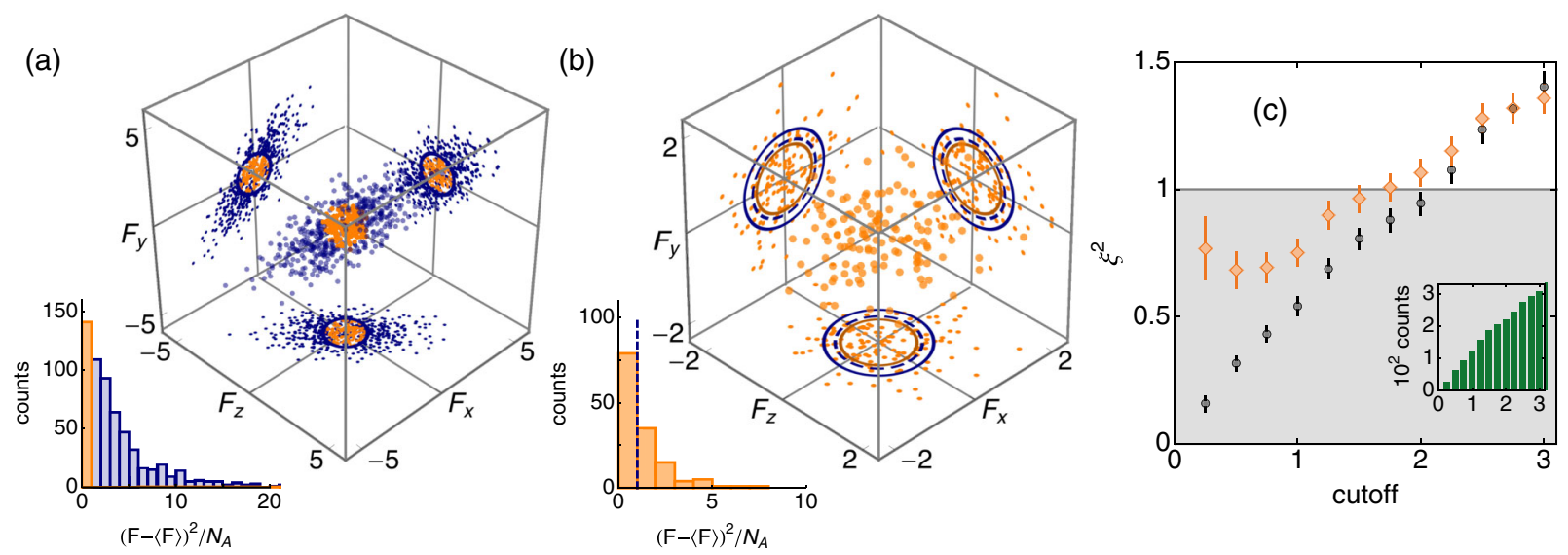

FIG. 2 (color online). Selection of a macroscopic spin singlet. From the initial spin distribution [blue data in (a)], we select data with $\left|\hat{\mathbf{F}}^{(1)}-\left\langle\hat{\mathbf{F}}^{(1)}\right\rangle\right|^{2} / N_{A}<C$ [orange data in (a)], where $C$ is a chosen cutoff parameter. We then analyze the second QND measurement $\hat{\mathbf{F}}^{(2)}$ of the same data [orange data in (b)] to detect spin squeezing and entanglement. We illustrate this with data from a sample with $N_{A}=1.1 \times 10^{6}$ atoms and $C=1$. Axes in (a) and (b) have units of $10^{3}$ spins. In (a), the solid blue circle has a radius $\sqrt{C N_{A}}$. In (b), the solid blue circles have a radius equal to the $1 \sigma$ Gaussian rms of an input ideal TSS with $\xi^{2}=2$, including detection noise, and the dashed blue circles the same for a state with $\xi^{2}=1$. The solid orange circles in (b) indicates the measured $1 \sigma$ Gaussian rms of the selected data. In the insets, we plot a histogram of the first and second measurements. The selected data are plotted in orange, and the dashed blue line in (b) indicates the cutoff. (c) Spin-squeezing parameter $\xi^{2}$ (orange diamonds) calculated from the second QND measurement of the selected data as a function of the cutoff parameter $C$. The shaded gray region indicates $\xi^{2}<1$, i.e., spin squeezing according to the criterion given in Eq. (1). For reference, the same parameter calculated from the first QND measurement is also plotted (black circles). Inset: Number of selected data points included as a function of the cutoff parameter.

cycle, the measurement is repeated without atoms in the trap. The loading cycle is repeated 602 times to gather statistics.

To detect the MSS, we make two successive measurements of the collective spin vector $\hat{\mathbf{F}}$ for each state preparation. The first measurements give us a record of the input spin distribution [blue points in Fig. 2(a)]. The spread of these data includes contributions from technical noise in the atomic state preparation and readout noise in the detection system. We select from the first measurements the events near the mean [orange points in Fig. 2(a)], i.e., a low-dispersion subset of our data [42]. The second measurement of these selected events, shown in Fig. 2(b), is analyzed to determine if the selected subset satisfies the criterion for a MSS.

The selection procedure is illustrated in Figs. 2(a) and 2(b). We select data from the first QND measurement of the collective spin vector using the criterion $|\hat{\mathbf{F}}-\langle\hat{\mathbf{F}}\rangle|^{2} / N_{A}<C$, where $C$ is a chosen cutoff parameter. We calculate $\xi^{2}=\tilde{\mathcal{V}}_{2} /\left(f N_{A}\right)$ from the second QND measurement, where $\tilde{\mathcal{V}}_{2}$ is the total variance after subtraction of the readout noise $\tilde{\mathcal{V}}_{2} \equiv \mathcal{V}_{2}-\mathcal{V}_{0}$. Here, $\mathcal{V}_{2} \equiv \operatorname{Tr}\left(\Gamma_{2}\right)$, where $\Gamma_{2}$ is the covariance matrix corresponding to the second QND measurement, and the readout noise $\mathcal{V}_{0} \equiv \operatorname{Tr}\left(\Gamma_{0}\right)$ is quantified by repeating the measurement without atoms in the trap and calculating the corresponding covariance matrix $\Gamma_{0}$. For this experiment, $\mathcal{V}_{0}=9.2 \pm 0.3 \times 10^{5}$ spins $^{2}$. This selection procedure is a form of measurement-induced spin squeezing [21], verified by the second QND measurement. In Fig. 2(c), we show $\xi^{2}$, computed on the second measurements of the selected events, as a function of the cutoff parameter $C$ for data from a sample with $N_{A}=1.1 \times 10^{6}$. With a cutoff $C=0.75$, we measure $\xi^{2}=0.69 \pm 0.05$, detecting entanglement with $5 \sigma$ significance.

We cross-check our results by repeating the experiment under near-identical conditions and analyzing the conditional covariance between successive vector spin measurements. This allows us to deterministically prepare a MSS without filtering our data. For these measurements, the applied magnetic field had a magnitude $B=15.9 \mathrm{mG}$, giving a Larmor period of $T_{L}=90 \pm 3 \mu \mathrm{s}$, and we repeated the experiment 155 times.

Correlations between successive measurements of the same spin component $\hat{F}_{k}$ allow us to predict the outcome of the second measurements $F_{k}^{(2)}$ with a reduced conditional uncertainty. For a single parameter, the conditional variance is $\operatorname{var}\left(F_{k}^{(2)} \mid F_{k}^{(1)}\right) \equiv \operatorname{var}\left(F_{k}^{(2)}-\chi F_{k}^{(2)}\right)$, where the correlation parameter $\chi \equiv \operatorname{cov}\left(F_{k}^{(1)}, F_{k}^{(2)}\right) / \operatorname{var}\left(F_{k}^{(1)}\right)$ minimizes the conditional variance [21]. This is illustrated in Fig. 3.

This procedure is readily extended to the conditional covariance using standard multivariate statistics. We calculate the total variance $\mathcal{V}_{1,2} \equiv \operatorname{Tr}\left(\Gamma_{1,2}\right)$ of the QND measurements, where $\left(\Gamma_{c}\right)_{i j} \equiv \operatorname{cov}\left(\hat{F}_{i}^{(c)}, \hat{F}_{j}^{(c)}\right) \equiv \frac{1}{2}\left\langle\hat{F}_{i}^{(c)} \hat{F}_{j}^{(c)}+\right.$ $\left.\hat{F}_{j}^{(c)} \hat{F}_{i}^{(c)}\right\rangle-\left\langle\hat{F}_{i}^{(c)}\right\rangle\left\langle\hat{F}_{j}^{(c)}\right\rangle$. Conditional noise reduction is quantified via $\mathcal{V}_{2 \mid 1}=\operatorname{Tr}\left(\Gamma_{2 \mid 1}\right)$, i.e., the total variance of the 


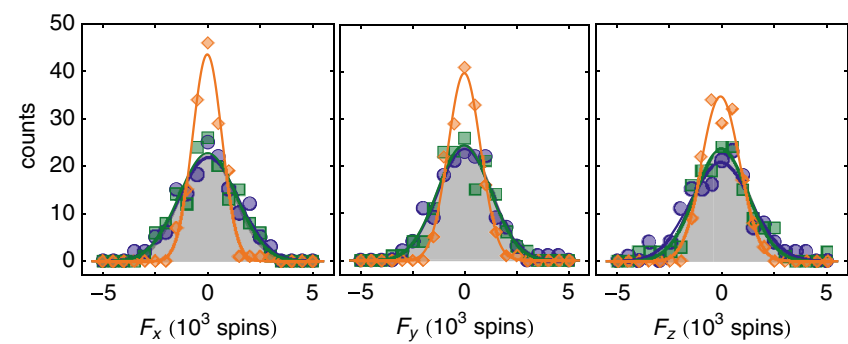

FIG. 3 (color online). Individual spin measurements. Histograms of the measurements of each of the three spin components are shown in the first round (blue circles) and second round (green squares) of the stroboscopic probe. We also show the conditional spin distribution $F_{k}^{(2)}-\chi F_{k}^{(1)}$ (orange diamonds), where $\chi \equiv \operatorname{cov}\left(F_{k}^{(1)}, F_{k}^{(2)}\right) /\left(\Delta F_{k}^{(1)}\right)^{2}$ is the degree of correlation. The shaded gray regions indicate the expected distribution for an ideal input TSS, including detection noise. For presentation purposes, an offset (between 5 and $10 \times 10^{3}$ spins) has been subtracted from the data (see the Supplemental Material [35])

conditional covariance matrix $\Gamma_{2 \mid 1} \equiv \Gamma_{2}-\Gamma_{2,1} \Gamma_{1}^{-1} \Gamma_{1,2}$, where $\Gamma_{1,2} \equiv \operatorname{cov}\left(\hat{F}_{i}^{(1)}, \hat{F}_{j}^{(2)}\right)$ [43]. To estimate the atomic noise contribution, we fit the polynomial $\mathcal{V}_{\alpha}\left(N_{A}\right)=\mathcal{V}_{0}+$ $2 N_{A}+c N_{A}^{2}$ to the measured data for the two QND measurements and the conditional variance (see the Supplemental Material [35]). We then calculate $\tilde{\mathcal{V}}_{\alpha}=\mathcal{V}_{\alpha}-\mathcal{V}_{0}$, subtracting the readout noise from the measured total variances.

In Fig. 4(a), we plot $\tilde{\mathcal{V}}_{1,2}\left(N_{A}\right)$, the total measured variance as a function of the number of atoms in the trap for the first two QND measurements (blue circles and green squares). An ideal TSS has a total variance $\tilde{\mathcal{V}}=\left\langle F^{2}\right\rangle-$ $\langle F\rangle^{2}=2 N_{A}$ [solid black line in Fig. 4(a)]. Because of technical noise contribution, the measured variances are higher than the ideal variance for TSS. The technical noise contribution to $\tilde{\mathcal{V}}_{1}$ is indicated by the upper shaded blue region. A conditional variance $\tilde{\mathcal{V}}_{2 \mid 1}<f N_{A}$ (lower shaded gray region) indicates spin squeezing and detects entanglement among the atoms $[23,28-30]$. The measured conditional variance $\tilde{\mathcal{V}}_{2 \mid 1}$ (orange diamonds) indicates that we produce spin squeezed states for $N_{A}>5 \times 10^{5}$ atoms. The conditional noise for an ideal QND measurement is $\tilde{\mathcal{V}}_{2 \mid 1}=2 N_{A} /(1+\zeta)$, where $\zeta=\frac{2}{3} G_{1}^{2} N_{L} N_{A}$ is the SNR of the measurement [21,38]. A fit to our data (orange dot-dashed line) gives $\tilde{\mathcal{V}}_{2 \mid 1}=2 N_{A} /(1+b \zeta)$, with $b=0.75 \pm 0.1$, where the reduction in SNR is due to technical noise in the detection system. In the inset of Fig. 4(a), we show the calculated spin-squeezing parameter $\xi^{2}=\tilde{\mathcal{V}}_{2 \mid 1} / f N_{A}$. With $N_{A}=1.1 \times 10^{6}$ atoms, we measure $\xi^{2}=0.50 \pm 0.09$, or $3 \mathrm{~dB}$ of spin squeezing detected with $5 \sigma$ significance. This level of squeezing implies that at least $5.5 \times 10^{5}$ atoms are entangled with at least one other atom in the ensemble [23]. While multipartite entanglement may also be generated in the ensemble [44], it is not detected by our spin-squeezing inequality [45].

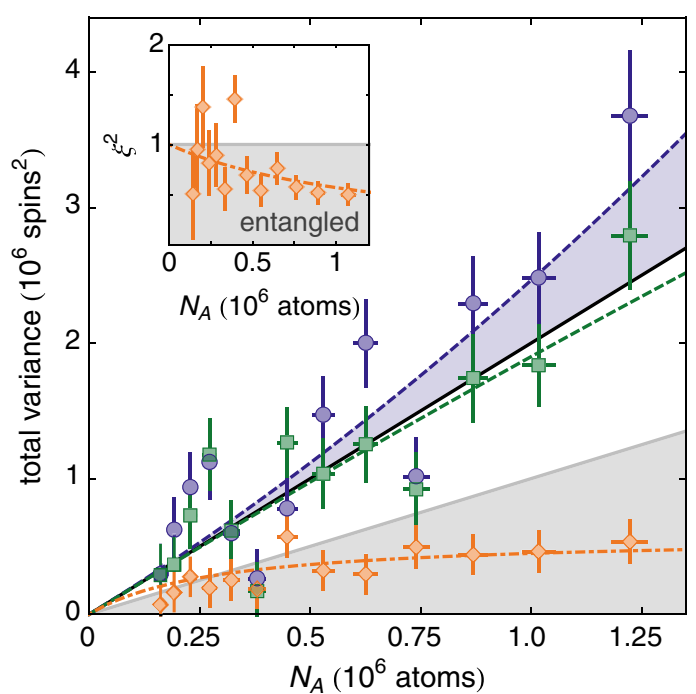

FIG. 4 (color online). Noise scaling of total variance $\tilde{\mathcal{V}}\left(N_{A}\right)$ of the first (blue circles) and second (green squares) QND measurements of the atomic spin distribution and of conditional variance $\tilde{\mathcal{V}}_{2 \mid 1}$ (orange diamonds). Dashed lines are a quadratic fit, indicating the presence of technical noise in the input atomic state (upper shaded blue region). The solid black line indicates the expected quantum noise $\tilde{\mathcal{V}}=2 f N_{A}$ due to an ideal TSS. The lower shaded gray region represents a region with $\tilde{\mathcal{V}}_{2 \mid 1}<f N_{A}$, indicating spin squeezing and entanglement. The dot-dashed orange curve is a fit to the expected conditional noise reduction with the SNR of the QND measurement as a free parameter. Inset: Semilog plot of the detected spin-squeezing parameter. The dot-dashed curve is the expected spin squeezing calculated from the fitted SNR. Horizontal and vertical error bars represent $1 \sigma$ statistical errors, and readout noise has been subtracted from the data (see the Supplemental Material [35]).

We have demonstrated the conditional preparation of a MSS via stroboscopic QND measurement in an unpolarized ensemble with more than $10^{6}$ laser-cooled atoms. We observe $3 \mathrm{~dB}$ of spin squeezing and detect entanglement with $5 \sigma$ statistical significance using a generalized spinsqueezing inequality, indicating that at least half the atoms in the sample have formed singlets [23,28-30]. Our techniques complement existing experimental methods [3-7] and can be readily adapted to measurements of quantum lattice gases [24,26] and spinor condensates [25]. In future work, we aim to combine our measurement with quantum control techniques [40] to produce an unconditionally squeezed macroscopic singlet centered at the origin [23] and to use our spatially extended MSS for magnetic field gradiometry [46]. Because of its $S U(2)$ invariance, the MSS is a good candidate for storing quantum information in a decoherence-free subspace [47] and for sending information independent of a reference direction [48].

This work was supported by the Spanish MINECO (Projects No. FIS2011-23520 and No. FIS2012-36673- 
C03-03), by the EU (Projects ERC StG AQUMET, ERC StG GEDENTQOPT, and CHIST-ERA QUASAR), by the Basque Government (Project No. IT4720-10), and by the OTKA (Contract No. K83858).

* Corresponding author. naeimeh.behbood@icfo.es

[1] M. Lewenstein, A. Sanpera, V. Ahufinger, B. Damski, A. Sen(De), and U. Sen, Adv. Phys. 56, 243 (2007).

[2] M. Lewenstein, A. Sanpera, and V. Ahufinger, Ultracold Atoms in Optical Lattices: Simulating Quantum Many-Body Systems (Oxford University Press, Oxford, England, 2012).

[3] S. Trotzky, Y.-A. Chen, U. Schnorrberger, P. Cheinet, and I. Bloch, Phys. Rev. Lett. 105, 265303 (2010).

[4] J. Simon, W. S. Bakr, R. Ma, M. E. Tai, P. M. Preiss, and M. Greiner, Nature (London) 472, 307 (2011).

[5] S. Nascimbène, Y.-A. Chen, M. Atala, M. Aidelsburger, S. Trotzky, B. Paredes, and I. Bloch, Phys. Rev. Lett. 108, 205301 (2012).

[6] D. Greif, L. Tarruell, T. Uehlinger, R. Jördens, and T. Esslinger, Phys. Rev. Lett. 106, 145302 (2011).

[7] D. Greif, T. Uehlinger, G. Jotzu, L. Tarruell, and T. Esslinger, Science 340, 1307 (2013).

[8] R. Islam, C. Senko, W. C. Campbell, S. Korenblit, J. Smith, A. Lee, E. E. Edwards, C.-C. J. Wang, J. K. Freericks, and C. Monroe, Science 340, 583 (2013).

[9] P. W. Anderson, Science 235, 1196 (1987).

[10] L. Balents, Nature (London) 464, 199 (2010).

[11] E. R. Livine and D. R. Terno, Phys. Rev. A 72, 022307 (2005).

[12] M. Koschorreck, M. Napolitano, B. Dubost, and M. W. Mitchell, Phys. Rev. Lett. 104, 093602 (2010).

[13] M. Koschorreck, M. Napolitano, B. Dubost, and M. W. Mitchell, Phys. Rev. Lett. 105, 093602 (2010).

[14] R. J. Sewell, M. Napolitano, N. Behbood, G. Colangelo, and M. W. Mitchell, Nat. Photonics 7, 517 (2013).

[15] A. Kuzmich, N. P. Bigelow, and L. Mandel, Europhys. Lett. 42, 481 (1998).

[16] J. Appel, P. J. Windpassinger, D. Oblak, U. Hoff, N. Kjaergaard, and E. S. Polzik, Proc. Natl. Acad. Sci. U.S.A. 106, 10960 (2009).

[17] T. Takano, M. Fuyama, R. Namiki, and Y. Takahashi, Phys. Rev. Lett. 102, 033601 (2009).

[18] M. H. Schleier-Smith, I. D. Leroux, and V. Vuletić, Phys. Rev. Lett. 104, 073604 (2010).

[19] I. D. Leroux, M. H. Schleier-Smith, and V. Vuletić, Phys. Rev. Lett. 104, 073602 (2010).

[20] Z. Chen, J. G. Bohnet, S. R. Sankar, J. Dai, and J. K. Thompson, Phys. Rev. Lett. 106, 133601 (2011).

[21] R. J. Sewell, M. Koschorreck, M. Napolitano, B. Dubost, N. Behbood, and M. W. Mitchell, Phys. Rev. Lett. 109, 253605 (2012).

[22] J. G. Bohnet, K. C. Cox, M. A. Norcia, J. M. Weiner, Z. Chen, and J. K. Thompson, arXiv:1310.3177 [Nat. Photon. (to be published)].

[23] G. Tóth and M.W. Mitchell, New J. Phys. 12, 053007 (2010).
[24] K. Eckert, O. Romero-Isart, M. Rodriguez, M. Lewenstein, E. S. Polzik, and A. Sanpera, Nat. Phys. 4, 50 (2008).

[25] K. Eckert, L. Zawitkowski, A. Sanpera, M. Lewenstein, and E. S. Polzik, Phys. Rev. Lett. 98, 100404 (2007).

[26] P. Hauke, R. J. Sewell, M. W. Mitchell, and M. Lewenstein, Phys. Rev. A 87, 021601 (2013).

[27] G. Tóth, Phys. Rev. A 69, 052327 (2004).

[28] G. Tóth, C. Knapp, O. Gühne, and H. J. Briegel, Phys. Rev. Lett. 99, 250405 (2007).

[29] G. Tóth, C. Knapp, O. Gühne, and H. J. Briegel, Phys. Rev. A 79, 042334 (2009).

[30] G. Vitagliano, P. Hyllus, I. L. Egusquiza, and G. Tóth, Phys. Rev. Lett. 107, 240502 (2011).

[31] G. Vitagliano, I. Apellaniz, I. L. Egusquiza, and G. Tóth, Phys. Rev. A 89, 032307 (2014).

[32] B. Lücke, J. Peise, G. Vitagliano, J. Arlt, L. Santos, G. Tóth, and C. Klempt, Phys. Rev. Lett. 112, 155304 (2014).

[33] T. S. Iskhakov, M. V. Chekhova, G. O. Rytikov, and G. Leuchs, Phys. Rev. Lett. 106, 113602 (2011).

[34] T. S. Iskhakov, I. N. Agafonov, M. V. Chekhova, G. O. Rytikov, and G. Leuchs, Phys. Rev. A 84, 045804 (2011).

[35] See Supplemental Material at http://link.aps.org/ supplemental/10.1103/PhysRevLett.113.093601 for further details and supporting experiments.

[36] S. R. de Echaniz, M. Koschorreck, M. Napolitano, M. Kubasik, and M.W. Mitchell, Phys. Rev. A 77, 032316 (2008).

[37] G. Colangelo, R. J. Sewell, N. Behbood, F. M. Ciurana, G. Triginer, and M. W. Mitchell, New J. Phys. 15, 103007 (2013).

[38] K. Hammerer, K. Mølmer, E. S. Polzik, and J. I. Cirac, Phys. Rev. A 70, 044304 (2004).

[39] N. Behbood, F. M. Ciurana, G. Colangelo, M. Napolitano, M. W. Mitchell, and R. J. Sewell, Appl. Phys. Lett. 102, 173504 (2013).

[40] N. Behbood, G. Colangelo, F. Martin Ciurana, M. Napolitano, R. J. Sewell, and M. W. Mitchell, Phys. Rev. Lett. 111, 103601 (2013).

[41] M. Kubasik, M. Koschorreck, M. Napolitano, S. R. de Echaniz, H. Crepaz, J. Eschner, E.S. Polzik, and M. W. Mitchell, Phys. Rev. A 79, 043815 (2009).

[42] T. Fukuhara, A. Kantian, M. Endres, M. Cheneau, P. Schausz, S. Hild, D. Bellem, U. Schollwock, T. Giamarchi, C. Gross, I. Bloch, and S. Kuhr, Nat. Phys. 9, 235 (2013).

[43] M. Kendall and A. Stuart, The Advanced Theory of Statistics (Griffin, London, 1979), Vol. 2.

[44] I. Urizar-Lanz, Ph.D. thesis, Universidad del Pais Vasco, 2014.

[45] Multipartite entanglement can also be detected by generalized spin-squeezing inequalities. See, for example, Ref. [32] and J. K. Korbicz, J. I. Cirac, and M. Lewenstein, Phys. Rev. Lett. 95, 120502 (2005).

[46] I. Urizar-Lanz, P. Hyllus, I. L. Egusquiza, M. W. Mitchell, and G. Tóth, Phys. Rev. A 88, 013626 (2013).

[47] D. A. Lidar, I. L. Chuang, and K. B. Whaley, Phys. Rev. Lett. 81, 2594 (1998).

[48] S. D. Bartlett, T. Rudolph, and R. W. Spekkens, Phys. Rev. Lett. 91, 027901 (2003). 